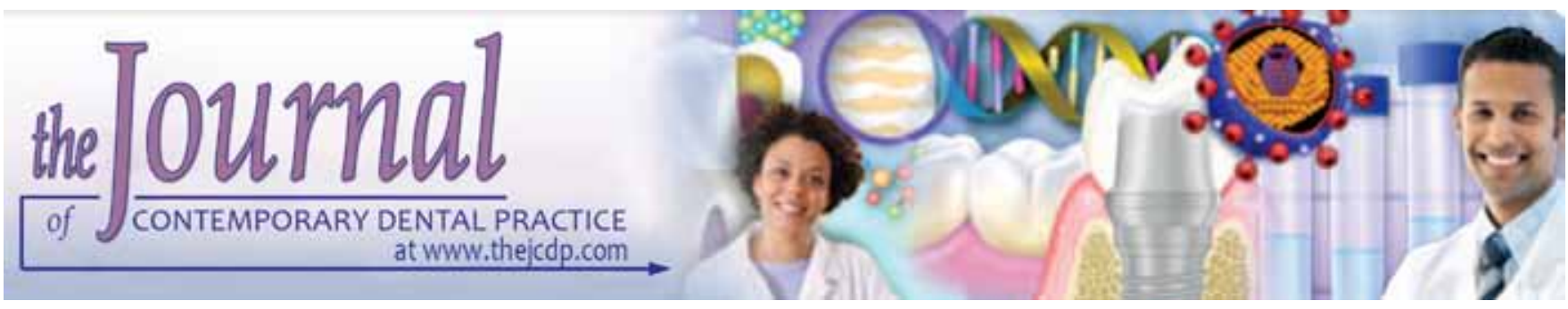

\title{
Effect of a Metal Primer on the Adhesive Interface between Composite and Lingual Brackets
}

Julio Pedra e Cal Neto, José de Albuquerque Calasans-Maia, Natalia Valli de Almeida, Helen Rohen

Maria Angélica Galvão Freire

\section{ABSTRACT}

Aim: To test the null hypothesis was that there is no difference in the shear strength of indirectly bonded lingual brackets with or without prior application of metal primer on their bases.

Materials and methods: Forty recently extracted human premolars were obtained and randomly divided into two groups of 20 each: group I (control), phosphoric acid and indirect bonding with Maximum Cure (Reliance Orthodontic Products, Itasca, IL, USA); and group II, application of metal primer (Metal Primer, Reliance Orthodontic Products) on bracket base prior to conditioning and indirect bonding. All products were used according to the manufacturer's instructions. A universal testing machine was used to apply a shear force directly onto the enamel- bracket interface at a speed of $1.0 \mathrm{~mm} / \mathrm{min}$.

Results: Mean (SD) shear bond strength for group I was 12.87 (5.75) MPa and for the group II was 18.47 (8.48) MPa. The Student's t-test showed a significant difference $(p=0.0311)$ between the groups. The chi-square test for the adhesive remnant index $(A R I)$ indicated that there was a significant difference $(p=0.2750)$.

Conclusion: The application of metal primer increased the adhesion of lingual brackets, and may be a promising procedure for clinical orthodontics.

Keywords: Temporomandibular joint dysfunction, Prevalence, edentulous, North Gujarat, Clicking sounds, Masseter.

How to cite this article: e Cal Neto JP, de Albuquerque Calasans-Maia J, de Almeida NV, Rohen H, Freire MAG. Effect of a Metal Primer on the Adhesive Interface Between Composite and Lingual Brackets. J Contemp Dent Pract 2013;14(6):11061108

\section{Source of support: $\mathrm{Nil}$}

Conflict of interest: None declared

\section{INTRODUCTION}

Several improvements in materials and laboratory processes are increasing the attractiveness of the lingual technique. However, the adhesive failure of brackets remains a limitation during the treatment, especially in adults with different restorations. ${ }^{1}$

Some adhesion boosters containing 4-META have been presented with the purpose to increase adhesion to dental alloys. ${ }^{2-4}$ In amalgam bonding, one of the most commonly used intermediate resins containing 4-META is Reliance Metal Primer (MP; Reliance, Itasca, Illinois, USA). With MP use, was demonstrated the increase of adhesive strength of composite to amalgam ${ }^{3,4}$ and the efficacy in preventing infiltration of oral fluid between metal and resin. ${ }^{5}$

It was assumed that applying MP on the base of the metal bracket would improve the superficial energy at the bracket base and increase the adhesion between the composite and bracket. The aim of this study was to investigate the effect of a MP on the adhesive interface between composite and lingual brackets bonded indirectly. The null hypothesis was that there is no difference in shear strength of lingual appliances bonded with or without prior application of MP on their bases.

\section{MATERIALS AND METHODS}

A sample size of 28 brackets $(n=14)$ would be sufficient to detect a difference of $5 \mathrm{MPa}$ between groups, with $80 \%$ power and a 5\% significance level (StatMate 2.0, GraphPad Software, San Diego, Calif). A total of 40 human premolars free from caries, cracks and restorations were used. Ethical approval was obtained for collection of the teeth from the State University of Rio de Janeiro Research Ethics Committee.

Lingual orthodontic maxillary premolar brackets (Tecnident, São Carlos, SP, Brazil) were used in this study. The average bracket base surface area was $9.42 \mathrm{~mm}^{2}$. The indirect bonding technique was performed in the following manner: the lingual surfaces of the teeth were painted with separating medium (Cel-lac, SS White, Rio de Janeiro, RJ, Brazil), and then dried with compressed air free from oil for 
5 seconds. The bracket base was cleaned with acetone for 5 seconds. The teeth were randomly assigned into two groups of 20 specimens, and the brackets were prepared on the lingual surfaces according to the manufacturer's instructions following one of the two protocols:

Group I (control) - Transbond XT adhesive paste (3M Unitek, Monrovia, CA, USA) was applied to the bracket to form the custom composite base, and then pressed firmly on the lingual surfaces of the tooth. Excess adhesive was removed with a small scaler, and the brackets were light cured positioning the light guide of an Ortholux XT Visible light-curing unit (3M Unitek) for 20 seconds.

Group II (MP): The chemically cured MP (Reliance Metal Primer) was applied on the base of the brackets prior to the application of the Transbond XT adhesive paste as described in group I.

In both groups, transfer trays were made from acrylic resin (Duralay, Reliance) for each tooth. The transfer trays with the brackets were removed from the teeth. The composite adhesive on the custom bracket base was cleaned with acetone for 5 seconds.

After cleaning the teeth with a rubber cup and pumice for 5 seconds, they were rinsed with water spray and dried with compressed air for an additional 5 seconds. The teeth were etched with $37 \%$ phosphoric acid gel (Reliance) for 30 seconds, then rinsed thoroughly with water for 30 seconds, and completely dried with compressed oil-free air. Maximum Cure (Reliance) was used to bond the custom bracket bases to the lingual surfaces of the teeth.

After bonding was completed, the transfer trays were removed. The specimens were mounted in plastic rings with acrylic. A mounting jig was used to align the bracket base to be perpendicular with the bottom of the mold and parallel to the force during the shear strength test.

Universal testing machine (EMIC DL 200MF, São José dos Pinhais, PR, Brazil) was used to apply an occlusogingival load to the bracket, which produced a shear force at the toothbracket interface with a crosshead speed of $1 \mathrm{~mm} / \mathrm{min}$.

\begin{tabular}{lllll}
\multicolumn{5}{|c}{ Table 1: Results of Student's t-test comparing shear bond } \\
strengths (MPa) of groups \\
\hline Groups & $N$ & Mean* & SD & Range \\
\hline I. Control & 20 & 12.87 & 5.75 & $6.15-24.29$ \\
II. Metal Primer & 20 & 18.47 & 8.48 & $7.73-35.25$ \\
\hline
\end{tabular}

${ }^{*} p=0.0311$

\begin{tabular}{|c|c|c|c|c|c|}
\hline \multirow[t]{2}{*}{ Groups } & \multirow[t]{2}{*}{$n$} & \multicolumn{4}{|c|}{ ARI Scores } \\
\hline & & 0 & 1 & 2 & 3 \\
\hline I. Control & 20 & 4 & 5 & 4 & 7 \\
\hline II. Metal primer & 20 & 2 & 11 & 3 & 4 \\
\hline Total & 40 & 6 & 16 & 7 & 11 \\
\hline
\end{tabular}

${ }^{*} p=0.2750 ; \chi^{2}=3.878$
After debonding, the teeth and brackets were examined to evaluate the amount of resin remaining on the tooth. The adhesive remnant index (ARI) score has a range between 0 and 3 as follows: 0 , no adhesive remained on the tooth; 1 , less than half of the enamel bonding site was covered with adhesive; 2 , more than half of the enamel bonding site was covered with adhesive; and 3 , the enamel bonding site was covered entirely with adhesive.

Descriptive statistics were calculated for each group. The data of bond strength were tested for normality with D'Agostino \& Pearson method. The unpaired Student's t-test was used to determine whether significant differences were present between the two groups. All statistical analyses were performed with the software Prism 4.0 (GraphPad Software, San Diego, California, USA) at a 5\% level of significance.

\section{RESULTS}

The descriptive statistics comparing the shear strength of the two groups are given in Table 1. The unpaired Student's $\mathrm{t}$-test detected a significant difference $(\mathrm{p}=0.0311)$ between groups evaluated. The MP group had the highest mean debond value at $18.47 \mathrm{MPa}$, whereas the control group had the lowest value at $12.87 \mathrm{MPa}$ (Fig. 1).

The ARI scores for the two groups tested are listed in Table 2. The results of chi-square test comparisons for the ARI indicated that there were not significant differences between the groups $\left(p=0.2750, \chi^{2}=3.878\right)$. With the application of MP there was a higher frequency of ARI scores of 1 , which indicated that more composite remained on the bracket base, if compared with group 1 .

\section{DISCUSSION}

The null hypothesis was rejected. The results of this study indicated that the application of MP on the brackets base prior the composite significantly affects the shear bond strength of lingual appliances on the enamel surface.

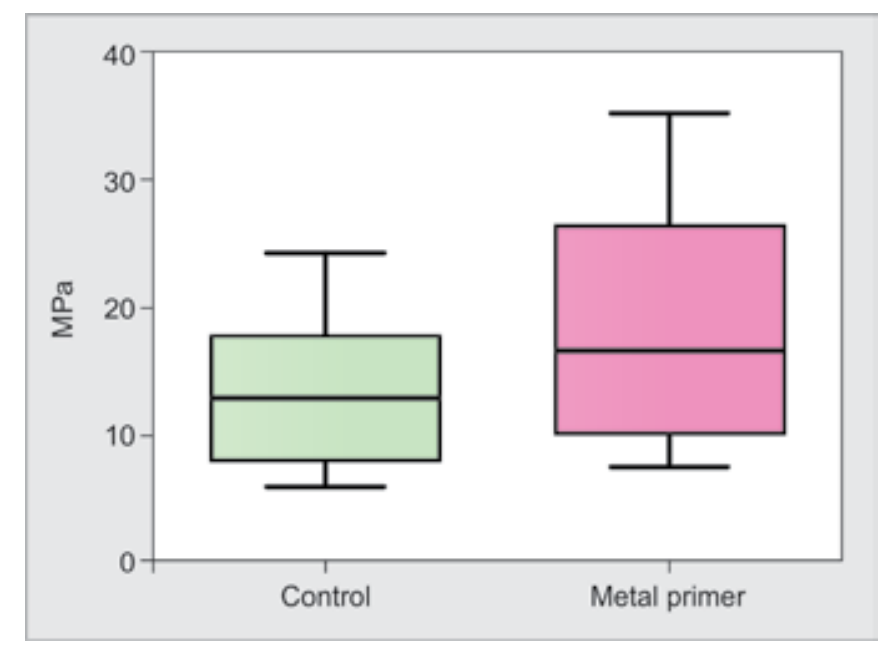

Fig. 1: Box plots for shear bond strength (in $\mathrm{MPa}$ ) of experimental groups 
Although there is not a formally accepted minimum clinical bond strength, the bond strength required to withstand normal orthodontic forces is believed to be between 8 and $9 \mathrm{MPa}^{6}$. In this study, the bracket failure occurred between 12.87 and $18.47 \mathrm{MPa}$. These results in agreement with other studies suggest that adequate bond strengths can be achieved when the application of MP is carried out on the brackets base prior the indirect bonding. ${ }^{3,4}$

The evaluation of the ARI scores indicated no significant difference in bond failure site between the two groups. However, the results showed that the MP group left less adhesive on enamel than the control group. This fact can be advantageous for clinicians when removing the adhesive after debonding brackets, making it simpler and faster. ${ }^{7}$

The application of MP seems to be a useful procedure in the routine of lingual orthodontics. However, this was a preliminary study and care should be taken in interpreting the results. To recommend the use of metal primers in large scale, further studies are required particularly in vivo studies and clinical trials. ${ }^{8,9}$

\section{CONCLUSION}

Under the conditions of this investigation, the metal primer application increases the shear bond strength of lingual brackets indirectly bonded.

\section{CLINICAL SIGNIFICANCE}

Bracket failures in lingual orthodontics are very undesirable. Metal primers can be very useful to increase the bond strength of them.

\section{ACKNOWLEDGMENT}

This investigation was supported in part by Research Grant APQ 1 from the Rio de Janeiro State Research Foundation (FAPERJ).

\section{REFERENCES}

1. Wiechmann D. Lingual Orthodontics (Part 3): Intraoral Sandblasting and Indirect Bonding. J Orofac Orthop 2000;61(4): 280-291.

2. Matsumura H, Tanaka T, Astuta M. Effect of acidic primers on bonding between stainless steel and auto-polymerizing methacrylic resins. J Dent 1997 May-Jul;25:285-290.
3. Gross MW, Foley TF, Mamandras AH. Direct bonding to Adlloytreated amalgam. Am J Orthod Dentofacial Orthop 1997 Sep; 112(3):252-258

4. Büyükyilmaz T, Zachrisson B. Improved orthodontic bonding to silver amalgam. Part 2. Lathe-cut, admixed, and spherical amalgams with different intermediate resins. Angle Orthod 1998 Aug;68(4):337-344

5. Yasuda N, Sasaki M, Mogi T, Ai M, Nakabayashi N. Influence between metal and resin on the finishing line of cobalt-chromium denture. Part 2. Effect of 4-META on preventing dye penetration at the finishing line. J Jpn Prosthodont Soc 1978;22:761-765.

6. Sunna S, Rock WP. Clinical performance of orthodontic brackets and adhesive systems: a randomized clinical trial. Br J Orthod 1998 Nov;25(4):283-287.

7. Vicente A, Bravo LA, Romero M. Influence of a nonrinse conditioner on the bond strength of brackets bonded with a resin adhesive system. Angle Orthod 2005 May;75(3):400-405.

8. Cal-Neto JP, Castro S, Moura PM, Ribeiro D, Miguel JAM. Influence of enamel sandblasting prior to etching on shear bond strength of indirectly bonded lingual appliances. Angle Orthod 2011 Jan;81(1):151-154.

9. Cal-Neto JP, Quintão CA, Almeida MA, Miguel JA. Bond failure rates with a self-etching primer: a randomized controlled trial. Am J Orthod Dentofacial Orthop 2009 Jun;135(6):782-786.

\section{ABOUT THE AUTHORS}

\section{Julio Pedra e Cal Neto (Corresponding Author)}

Professor, Division of Orthodontics, Fluminense Federal University Nova Friburgo, Rio de Janeiro, Brazil, Phone: 552225287168, e-mail: juliocalneto@yahoo.com.br

\section{José de Albuquerque Calasans-Maia}

Professor, Division of Orthodontics, Fluminense Federal University, Nova Friburgo, Rio de Janeiro, Brazil

\section{Natalia Valli de Almeida}

Former Resident, Division of Orthodontics, Fluminense Federal University, Niterói, Rio de Janeiro, American Samoa

\section{Helen Rohen}

Undergraduate Student, School of Dentistry, Fluminense Federal University, Nova Friburgo, Rio de Janeiro, Brazil

\section{Maria Angélica Galvão Freire}

Undergraduate Student, School of Dentistry, Fluminense Federal University, Nova Friburgo, Rio de Janeiro, Brazil 